\title{
Enhanced plasmid DNA delivery using anionic LPDII by listeriolysin 0 incorporation
}

\author{
Gretchen L. Lorenzi* \\ Kyung-Dall Lee
}

Department of Pharmaceutical Sciences, University of Michigan, Ann Arbor, MI 48109-1065, USA

*Correspondence to:

Gretchen L. Lorenzi, 1411 Broadway Street, Ann Arbor, MI 48105, USA.

E-mail: kdlee@umich.edu; gretchen@fulbrightweb.org
Received: 17 October 2004

Revised: 23 November 2004

Accepted: 13 December 2004

\begin{abstract}
Background A major obstacle to achieving effective DNA-based therapeutics is efficient delivery of the DNA to its site of action in the cell. Upon internalization by endocytosis, the endosomal membrane represents a critical physical barrier preventing access of DNA to the cell cytosol. In order to overcome the membrane barrier and facilitate cytosolic entry, the endosomolytic bacterial protein listeriolysin O (LLO) is a potentially promising agent.
\end{abstract}

Methods LLO was incorporated in an anionic liposome-entrapped polycation-condensed DNA delivery system (LPDII). Plasmid DNA was condensed using protamine sulfate and then complexed to anionic liposomes. LLO was incorporated into the delivery vehicle through encapsulation in anionic, $\mathrm{pH}$-sensitive liposomes. Transfection levels were monitored using a model reporter plasmid encoding luciferase in P388D1 cells, a macrophage-like cell line.

Results Transfection using the anionic LPDII delivery platform was enhanced through incorporation of LLO. Additionally, the net charge of the condensate, the lipid composition, and the total amount of LLOliposomes were all capable of modulating the transfection levels of the vehicle. Importantly, in the presence of serum, transfection levels using the LLO-containing LPDII system were comparable to established cationic lipid delivery systems.

Conclusions LLO is capable of facilitating transfection using an anionic LPDII system. This anionic delivery vehicle represents the successful combination of the LPDII system for condensation of the DNA with the unique endosomolytic properties of LLO for improved transfection using plasmid DNA. Copyright @ 2005 John Wiley \& Sons, Ltd.

Keywords listeriolysin O; protamine; LPDII; DNA delivery; liposome

\section{Introduction}

With the expansion of information arising from advances in genomics, new applications for gene therapy are being conceptualized increasingly rapidly. The field of gene delivery must continually strive to develop necessary technologies meeting the specific needs for the array of gene therapy protocols. While some gene delivery technologies, including both viral and non-viral vectors such as cationic liposomes and polymer-based delivery systems, have already shown great promise, each system has unique limitations [1-5]. Therefore, promising delivery concepts that build on 
existing, successful technologies must continue to be developed and refined.

Physical barriers to the cytosolic and nuclear delivery of genes represent key obstacles to the success of gene therapy. DNA delivery encompasses multiple steps in series: delivery to the specific tissue or cell target, passage across the cell membrane to gain cytosolic access, and transport into the nucleus $[4,6]$. Many innovative approaches are being taken to overcome each barrier in this multistep process of delivery. Often these individual solutions are in some respects modular; development of a specific functionality to overcome one barrier can be used in combination with those developed for preceding or subsequent barriers. For example, various membrane-disrupting components incorporated in both targeted polyplex and lipoplex gene delivery systems can significantly increase transfection levels beyond each system alone [7]. Thus, the focused development of technologies aimed at specific barriers, when used in combination, can lead to overall progress of successful DNA delivery technologies.

Delivery across the plasma membrane and into the cytosol of cells is one critical barrier to effective gene delivery $[8,9]$. The large size and anionic nature of plasmid DNA (pDNA) make it membrane-impermeant. Thus, naked pDNA is typically degraded extracellularly or taken up by cells and degraded in the endolysosomal compartment without ever reaching the cytosol. In the study reported in this paper, we investigated the combination of two promising technologies: anionic LPDII particles and listeriolysin O (LLO)-mediated cytosolic delivery. LPDII, a liposome-entrapped polycation-condensed pDNA delivery vehicle, utilizes anionic liposomes to envelop pDNA that has been condensed using polycations [10]. In the LPDII delivery system model of self-assembly, pDNA is first condensed with a polycation and this condensate is then electrostatically complexed, or entrapped, within liposomes [10]. While LPDII provides a platform upon which to build, the relatively low transfection efficiency of the anionic LPDII particle calls for further modifications, such as the addition of a targeting moiety or an endosomolytic agent.

To expand the utility and improve the efficiency of the LPDII particle, we have explored the incorporation of an additional functionality, an endosomolytic poreforming protein from the facultative intracellular bacteria, Listeria monocytogenes, listeriolysin O (LLO), to form an LLO-LPDII complex, and investigated its effect on pDNA delivery. To this end, LLO was encapsulated inside the anionic liposomes of LPDII for co-transport with the condensed pDNA complex. LLO has been demonstrated to be capable of breaching the endosomal membrane, allowing escape of endosomal or phagosomal contents into the cytosol of cells [11]. Furthermore, the activity of LLO is elevated and optimal at pH 5-6 of endosomes, making it ideal for applications where endosomal release is required $[9,12]$.

The versatility of LLO as a component in a delivery system is clear. LLO has been engineered to deliver intact bacteria to the cytosol of a cell; genetically modified Bacillus subtilis which express LLO are capable of endosomal escape, thus LLO is sufficient to impart endosomolytic function [13]. Utilizing purified or recombinant LLO, enhanced macromolecular delivery has been demonstrated with dyes and model proteins using the LLO-mediated escape mechanism [11], and recent work has shown that LLO-containing liposomes encapsulating a model protein are capable of enhancing the $\mathrm{CD} 8^{+}$cytotoxic $\mathrm{T}$ lymphocyte-based immune response in immunized mice [14]. LLO is also capable of delivering antisense oligonucleotides that are co-encapsulated in liposomes for down-regulation of cell-surface proteins [15].

This study is the first exploration of a liposomebased, LLO-mediated delivery system for pDNA. We hypothesized that addition of LLO in the LPDII system would improve transfection efficiency by improving access of the pDNA to the cytosol. LPDII and LLO-LPDII particles were formed using a model pDNA encoding luciferase under the cytomegalovirus (CMV) promoter. In keeping with the flexibility afforded by the original LPDII particle, modifications to the original LPDII system also include the use of protamine sulfate as the condensing polycation rather than poly(L-lysine). Protamine sulfate has several advantages as a condensing agent, including its current use in clinical treatments, which has provided extensive data to suggest that protamine is nontoxic, biocompatible, and weakly immunogenic [16]. Anionic pH-sensitive liposomes serve as the exterior of the complex such that endosomal uptake will trigger destabilization of the liposome and release of the encapsulated LLO [17]. We demonstrate that the LPDII particle can be greatly enhanced as a transfection agent with the addition of liposome-encapsulated LLO.

\section{Materials and methods}

\section{Materials}

Protamine sulfate (grade III, Clupeine) and cholesterylhemisuccinate (CHEMS) were purchased from Sigma. Egg phosphatidylethanolamine (PE), egg phosphatidylcholine (PC), 1,2-dioleoyloxy-3-trimethylammonium propane (DOTAP), and dioleoylphosphatidylethanolamine (DOPE) were purchased from Avanti Polar Lipids, Inc. (Alabaster, AL, USA). Lipofectamine was purchased from Invitrogen (Carlsbad, CA, USA).

\section{Preparation of plasmid containing the luciferase gene}

Plasmid DNA was obtained from the plasmid pNGVL3 encoding firefly luciferase under the cytomegalovirus (CMV) promoter (a gift from Dr. Gary Nabel, Vaccine Research Center, National Institutes of Health, MD, USA). Plasmid DNA was harvested and purified from E. coli using the Qiagen Plasmid maxi kit (Qiagen, CA, USA). 
The purity and concentration of pDNA was determined spectrophotometrically using the absorbances at 280 and $260 \mathrm{~nm}$.

\section{LLO purification}

Recombinant LLO was produced from E. coli BL21(DE3) which had been transformed with pET29b containing the LLO gene, hly, modified to include a six-histidine tag (a gift from Dr. Daniel Portnoy, University of California, Berkeley, CA, USA) [18]. The LLO was purified using a Ni-NTA column (Qiagen). A $500 \mathrm{ml}$ culture generally produced $\sim 10 \mathrm{mg}$ of protein. LLO purity was analyzed by sodium dodecyl sulfate/polyacrylamide gel electrophoresis (SDS-PAGE) and Coomassie blue staining. Protein concentration was determined by BCA assay (Pierce, IL, USA) using a standard of bovine serum albumin (BSA). The activity of LLO was determined using an in vitro sheep red blood cells (RBCs) hemolysis assay as previously described [14]. Briefly, sheep RBCs (ICN Biomedicals, OH, USA) were suspended in HEPESbuffered saline (10 mM HEPES, $140 \mathrm{mM} \mathrm{NaCl}, \mathrm{pH}$ 7.4) at $10^{8}$ cells $/ \mathrm{ml}$. Known amounts of purified recombinant LLO were added to $2 \mathrm{ml}$ of suspended RBCs, and changes in the right-angle light scattering of RBCs undergoing lysis were monitored using a fluorometer (Jobin Yvon-Spex Instruments SA., NJ, USA). The excitation and emission monochromators were set to $590 \mathrm{~nm}$.

\section{Cell culture}

The murine macrophage P388D1 cell line was purchased from the American Type Culture Collection (ATCC, Manassas, VA, USA). Cells were cultured in RPMI-1640 (Invitrogen, Carlsbad, CA, USA) supplemented with 10\% fetal bovine serum (FBS), $100 \mathrm{U} / \mathrm{ml}$ penicillin, $100 \mathrm{U} / \mathrm{ml}$ streptomycin, $10 \mathrm{mM}$ HEPES, and $1 \mathrm{mM}$ sodium pyruvate (complete RPMI). Cells were grown at $37^{\circ} \mathrm{C}$ in a $5 \% \mathrm{CO}_{2}$ humidified atmosphere.

\section{Protamine-pDNA complex formation}

Protamine-pDNA complexes at theoretical charge ratios from $0.64 / 1$ to $2.2 / 1(+/-)$ were prepared by adding $6 \mu \mathrm{g}$ of pDNA in $100 \mu \mathrm{l}$ HEPES-buffered glucose (HBgluc, $10 \mathrm{mM}$ HEPES, $280 \mathrm{mM}$ glucose, $\mathrm{pH}$ 8.5) to varying amounts of protamine in $200 \mu \mathrm{l}$ HBgluc while rapidly vortexing. The pDNA was added to the excess protamine (excess is based on charge ratios) unless a negative overall charge ratio was desired. Theoretical charge ratios are calculated based on: $1 \mu \mathrm{g}$ DNA is equivalent to $3.1 \mathrm{nmol}$ negative charge, and $1 \mathrm{nmol}$ protamine sulfate contains 21 nmol positive charge. The charge on the protamine-DNA condensate was monitored by dynamic light scattering in a zeta-potential/particle sizer instrument (NICOMP 380ZLS). As previously shown, pDNA condensed with increasing amounts of protamine displayed a neutral zeta-potential at a theoretical charge ratio of 1.25 amino groups in protamine to phosphates of pDNA [19]. Thus, all protamine-DNA condensates are reported here using theoretical charge ratios, with condensates formed at a theoretical charge ratio higher than 1.25/1 $(+/-)$ exhibiting a positive zeta-potential. Particle size of protamine-DNA condensates was also monitored using the NICOMP 380ZLS equipped with an Avalanche photodiode detector. Representative mean diameter for condensates formed at $1.6 / 1(+/-)$ was $100.6 \pm 15.5 \mathrm{~nm}$. In agreement with previous work, condensates formed closer to the charge neutral point tended to aggregate $(175 \pm 21.7 \mathrm{~nm})[10,19]$. Condensates with a net positive charge were able to occlude ethidium bromide (EtBr) in agarose gel analysis (data not shown). Briefly, pDNA was mixed with protamine sulfate at varying ratios as described above. A 0.8\% agarose gel was prepared $(0.4 \mathrm{~g}$

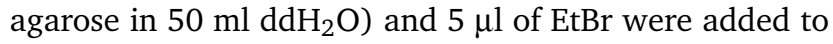
the solution. After the samples had been loaded, the gel was run at $100 \mathrm{~V}$ for $15 \mathrm{~min}$. The gel was then immediately analyzed under UV light using a Kodak digital camera and Metamorph Image analysis software. A polyacrylamide gel (20\%) was run in parallel with the same samples loaded in order to confirm, through protein staining using Coomassie blue, the presence of samples which did not display UV fluorescence in the agarose gel due to $\mathrm{EtBr}$ occlusion. Lack of fluorescence of $\mathrm{EtBr}$ in the presence of pDNA reflects an inability of the EtBr to intercalate into the pDNA due to condensation.

\section{Liposome preparation}

Liposomes were prepared using thin film hydration and the freeze/thaw technique. Briefly, lipids dissolved in chloroform were added at a 2:1 mole ratio of either PE/CHEMS or PC/CHEMS, and dried in a rotary evaporator under a stream of argon to a thin film. The lipid film was hydrated using $1 \mathrm{ml}$ of $3-$ fold diluted HBgluc (hypotonic HBgluc), either with or without $200 \mu \mathrm{g}$ LLO, and vortexed. The liposomes were subjected to five freeze/thaw cycles in an ethanol bath at $-80^{\circ} \mathrm{C}$ and sonicated twice for $30 \mathrm{~s}$ in a bath-type sonicator (Laboratory Supplies Company, NY, USA) [20]. Liposomes were then passed through a Sepharose CL4B gel filtration column (Amersham Pharmacia Biotech) equilibrated with HBgluc to remove unencapsulated LLO. Encapsulation efficiency of LLO was generally $15-25 \%$ in both PE/CHEMS and PC/CHEMS formulations. Typical diameter of PE/CHEMS liposomes by dynamic light scattering was $186.4 \pm 40.5 \mathrm{~nm}$. Addition of LLO did not affect liposome size. The charge contribution from liposomes for complex formation was calculated using the concentration of CHEMS in the liposome preparation. CHEMS concentration was derived from experimental determination of phosphate content in the liposome preparation following gel filtration and the molar ratio of phospholipids and CHEMS (PE/CHEMS or PC/CHEMS, $2: 1$ ). 


\section{Formation of LPDII and LLO-LPDII complexes}

LPDII and LLO-LPDII complexes were formed based on methods previously developed for LPDII [10]. ProtaminepDNA condensates at a concentration of $20 \mu \mathrm{g} \mathrm{pDNA} / \mathrm{ml}$ in $300 \mu \mathrm{l}$ were added to an equal volume of liposomes, either blank or LLO-containing, to form LPDII or LLOLPDII particles, respectively. The complexes were then diluted into an equal volume of complete RPMI-1640 containing 10\% FBS for transfection. Typical diameter of the LLO-LPDII complex by dynamic light scattering was $212.4 \pm 46.9 \mathrm{~nm}$. Zeta-potential analysis of the protamine-pDNA condensate alone at a $1.6: 1$ positive-tonegative charge ratio was $7.08 \pm 0.57 \mathrm{mV}$. Zeta-potential measurements were $-11.9 \pm 4.3 \mathrm{mV}$ following addition of anionic liposomes to a net theoretical charge of $-1.9 \mathrm{nmol} / \mu \mathrm{g}$ pDNA.

\section{Transfections}

P388D1 cells were plated at a density of $1.5 \times 10^{5}$ cells per well in 24-well plates and cultured for $16-24 \mathrm{~h}$ prior to transfection. Cells were typically $\sim 70 \%$ confluent at the time of the experiment. In all transfection assays, $200 \mu \mathrm{l} /$ well of the transfection sample containing $2 \mu \mathrm{g}$ pDNA in 5\% serum-containing RPMI1640 were added dropwise and incubated with the cells for $4 \mathrm{~h}$. The transfection media was removed after $4 \mathrm{~h}$ and the cells were washed once and replaced with fresh serumcontaining RPMI complete media. Cells were assayed for gene expression after $72 \mathrm{~h}$. Assays were performed in triplicate.

\section{Other transfection agents}

A comparative study of transfection using several commonly used transfection agents was undertaken. Chloroquine, DOTAP/DOPE $(1: 1)$, or Lipofectamine was added to transfection mixtures containing $2 \mu \mathrm{g}$ DNA. For Lipofectamine, samples were prepared with $2 \mu \mathrm{g}$ pDNA and added to the cells according to the manufacturer's instructions (Invitrogen). DOTAP and DOPE were mixed at a $1: 1$ ratio and mixed with $2 \mu \mathrm{g}$ pDNA. In chloroquinemediated samples, pDNA was condensed according to the above procedure with protamine, and $80 \mu \mathrm{M}$ chloroquine was added directly to the transfection mixture. All samples were incubated for $4 \mathrm{~h}$, after which the samples were removed and replaced with fresh serum-containing media, and incubated for $72 \mathrm{~h}$. All samples were then assayed as described.

\section{Luciferase assay}

Transfection was measured by detecting luciferase production using a luciferase assay kit (Promega,
WI, USA). Briefly, the cells were lysed using $100 \mu \mathrm{l}$ Reporter lysis buffer (Promega) and subjected to one freeze/thaw cycle. The cell lysates were then collected and centrifuged at $14000 \mathrm{rpm}$ for $90 \mathrm{~s}$. Then, $20 \mu \mathrm{l}$ of the supernatant were assayed for luminescence with $100 \mu \mathrm{l}$ luciferase substrate (Promega) using an AutoLumat LB953 luminometer (EG\&G Berthold) for $10 \mathrm{~s}$. Readings were normalized for total cellular protein concentration in the extracted samples using a BCA assay (Pierce). BCA assays were performed following the manufacturer's protocol using a standard of BSA. Cellular protein recovery is reported as the protein recovery, as determined by BCA, of each sample as a percentage of the cellular protein recovery by BCA of a control sample that was not transfected with any DNA but which was otherwise treated identically to test samples.

\section{Statistical analysis}

All statistical analyses were performed using GraphPad Prism version 4.0 (GraphPad Software, San Diego, CA, USA). One-way analysis of variance (ANOVA) with Tukey's post-test was performed on all data sets unless otherwise indicated. Studies analyzed with two-way ANOVA used the Bonferroni post-test.

\section{Results}

\section{Transfection using LLO-LPDII particles}

To investigate whether addition of LLO to the LPDII system augments transfection, P388D1 cells were transfected with either cationic protamine-DNA (P-D) condensate, anionic LPDII particles, or anionic LLO-LPDII complexes (Figure 1). P388D1 cells, a macrophage-like cell line, represent an appropriate in vitro cell model as liposomal carriers are naturally cleared from the circulation in vivo by macrophages of the reticuloendothelial system $[21,22]$. The protamine-DNA condensates were complexed to anionic liposomes to a net negative charge. In order to investigate the effect of LLO activity, the LLOcontaining liposomes were heated at $75^{\circ} \mathrm{C}$ for $10 \mathrm{~min}$ prior to complex formation and compared as a control formulation; incubation at $75^{\circ} \mathrm{C}$ for $10 \mathrm{~min}$ is sufficient to abolish LLO activity as monitored by a hemolysis assay (data not shown). Additionally, the protamine-DNA condensate was complexed with blank anionic liposomes in the presence of free LLO and this LPDII with free LLO mixture was used to transfect cells. The data in Figure 1 show that incorporation of LLO in the LPDII complex improved transfection levels more than 30-fold over both LPDII without LLO and cationic protamine-condensed pDNA $(p<0.001)$. This enhancement of transfection upon the incorporation of LLO in LPDII was abrogated and reduced to the levels of protamine-DNA alone in the samples in which LLO-containing liposomes were heat-inactivated $(p<0.001)$. Furthermore, addition of free LLO to wells 


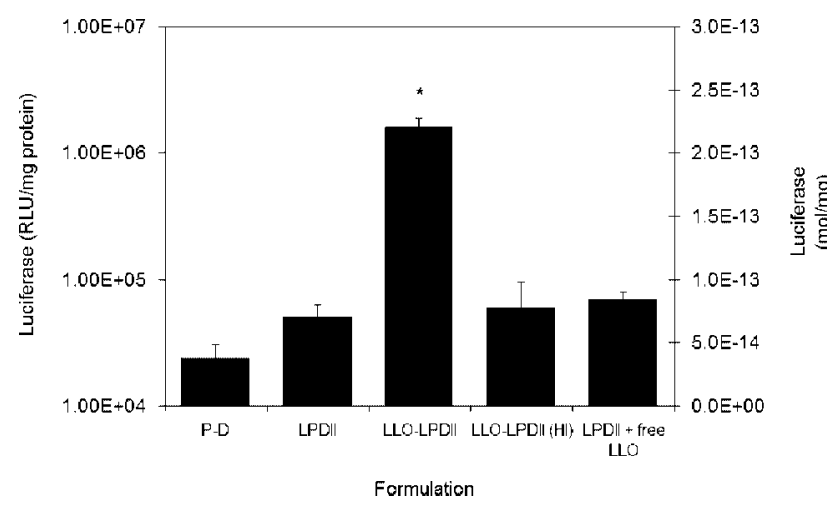

Figure 1. Effect of encapsulation of LLO in LPDII particles. pDNA was condensed with protamine at a 1.6:1 (+/-) theoretical charge ratio and administered either alone (P-D) or further complexed with PE/CHEMS liposomes. Complexes contained either blank liposomes (LPDII) or liposomes containing LLO (LLO-LPDII). Complexes were administered to P388D1 cells in transfection media containing 5\% FBS. As a control, LLO-LPDII complexes were formed using liposomes with LLO which had been heat-inactivated for $10 \mathrm{~min}$ at $75^{\circ} \mathrm{C}$ (LLO-LPDII (HI)). Also, LPDII were formed in the presence of $200 \mu \mathrm{g}$ in the $200 \mu \mathrm{l}$ transfection mixture (LPDII + free LLO). On the secondary axis, luciferase assay results were converted to luciferase concentration using a standard curve of known concentrations of luciferase enzyme. Results are reported as the mean \pm SEM of five experiments performed in triplicate. ${ }^{*} p<0.001$

transfected with LPDII did not significantly increase transfection over LPDII alone.

\section{Effects of protamine/pDNA ratio on LLO-LPDII transfection levels}

The effect of altering the protamine-to-pDNA ratio in the LLO-LPDII was determined by changing the amount of protamine while holding the amount of pDNA constant, thereby producing protamine-DNA condensates of varying net charge (Figure 2). Both anionic and cationic condensates were studied, with theoretical charge ratios of $0.64 / 1$ and $0.96 / 1(+/-)$ forming negatively charged condensates and ratios of $1.28 / 1,1.6 / 1,1.92 / 1$, and $2.24 / 1(+/-)$ forming positive condensates as verified by zeta-potential analysis (data not shown). All condensates were then mixed with LLO-containing anionic liposomes such that the theoretical net charge on the complexes was equivalent to $1.9 \mathrm{nmol}$ negative charge/ $\mu \mathrm{g}$ DNA. Anionic protamine-DNA condensates (those with $(+/-)$ ratios of $0.64 / 1$ and $0.94 / 1$ ) mixed with LLO-liposomes showed minimal transfection efficiency; transfection was comparable to protamine-DNA alone. All cationic protamine-DNA condensates (those with $+/-$ ratios of $1.28 / 1$ to $2.24 / 1$ ) which had been complexed to anionic liposomes showed 10- to 100-fold higher transfection levels than the anionic condensates $(p<0.05$ for all anionic vs. cationic condensates). Similarly, all cationic condensates showed significantly higher transfection than pDNA condensed with protamine at a 1.6/1 (+/-) charge ratio in the absence of LLO-liposomes $(p<0.05)$. Samples within each net charge grouping showed no significant differences.

\section{The effect of LLO-liposome/non-LLO- liposome (blank liposome) ratio}

The effect of the LLO content in the LLO-LPDII was examined by fixing the protamine, pDNA, and lipid content with the condensate charge ratio at $1.6 / 1$ $(+/-)$ and total lipid content at 1.9 nmol negative charge $/ \mu \mathrm{g}$ pDNA. Prior to addition to the protamineDNA condensate, liposomes were pre-mixed to specified ratios of blank to LLO-containing PE/CHEMS liposomes keeping the total amount of lipid fixed.

The data showed that as the percentage of LLOcontaining liposomes was decreased, the transfection level also decreased (Figure 3). The data showed an increase in gene expression with increasing incorporation of LLO-liposomes ( $p<0.001$ by one-way ANOVA with linear trend post-test). Inclusion of 50\% LLO-liposomes or higher was sufficient to significantly improve transfection over protamine-DNA and LPDII (equivalent to 0\% LLOliposomes; $p<0.05$ ).

\section{The effect of LLO-liposome/ protamine-DNA condensate ratio}

The next variable investigated while holding the amounts of protamine and pDNA constant was the total amount

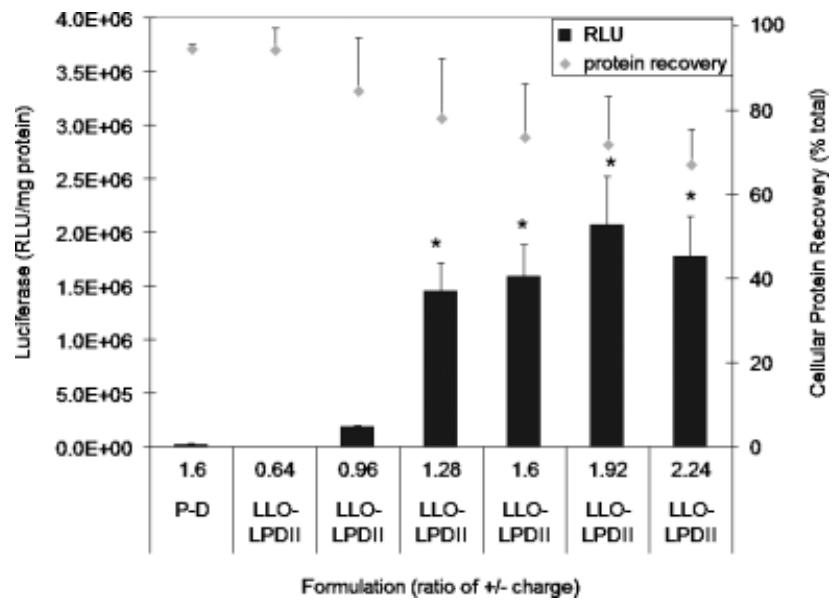

Figure 2. Effect of protamine-pDNA ratio in LLO-LPDII complexes on transfection activity. pDNA was condensed using various amounts of protamine sulfate to specific charge ratios based on the theoretical ratio of positively charged protamine to negatively charged DNA. The condensates were then complexed with anionic PE/CHEMS liposomes containing LLO to a net theoretical charge of $-1.9 \mathrm{nmol} / \mu \mathrm{g}$ pDNA. Protamine-pDNA condensate at a $1.6: 1(+/-)$ charge ratio was administered in the absence of liposomes as a control (P-D). Results are reported as the mean \pm SEM of three experiments performed in triplicate. ${ }^{*} p<0.05$ 


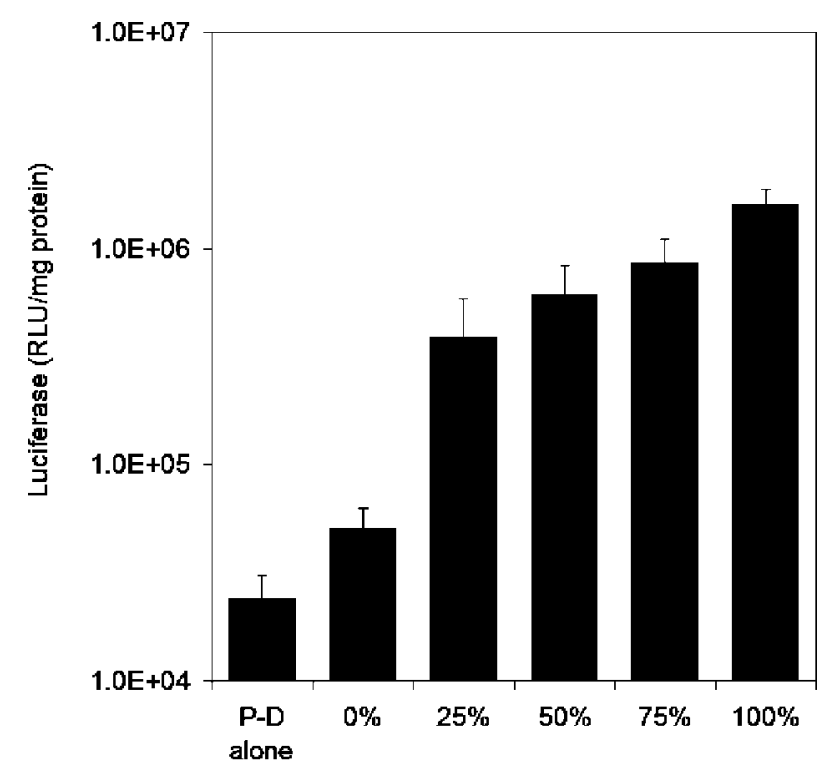

Lipid Formulation (\%LLO-containing liposomes)

Figure 3. Dependence of transfection activity on LLO content. Protamine-pDNA was condensed at a 1.6:1 (+/-) charge ratio and complexed to $\mathrm{PE} / \mathrm{CHEMS}$ liposomes to a net $-1.9 \mathrm{nmol}$ charge $/ \mu \mathrm{g}$ pDNA. The total amount of liposomes was kept constant as the percentage of those liposomes which encapsulated LLO was increased from all blank liposomes (0\%) to all LLO-containing liposomes (100\%). Complexes were administered in transfection media containing 5\% FBS. Results are reported as the mean \pm SEM of three experiments performed in triplicate. $p<0.001$ by one-way ANOVA with linear trend post-test

of LLO-containing liposomes in the LLO-LPDII system. We hypothesized that increasing the total amount of LLO-containing liposomes per protamine-DNA condensate would increase transfection efficiency. Formulations were composed of protamine-DNA at a 1.6/1 (+/-) charge ratio complexed to increasing amounts of LLO-containing liposomes (Figure 4). The lowest amount of liposomes used in Figure 4 corresponds to the theoretical isoelectric point of the complexes. As the amount of LLO-containing liposomes added was increased, however, the transfection efficiency was diminished. Addition of 10-fold more lipid reduced the transfection levels to those of protamine-DNA alone. Presence of excess LLO-liposomes also resulted in diminished recovery of total cellular protein, as monitored by BCA assay (Figure 4).

\section{Effect of pH-sensitivity of the liposomes}

LLO delivered in the LLO-LPDII complex is retained with the complex through encapsulation inside the enveloping liposomes. We hypothesized that the ability of LLO to facilitate endosomal release of pDNA is dependent on release of the LLO molecules from the liposomal encapsulation once the complex reaches the endosomes. To study this we used PC/CHEMS liposomes, a $\mathrm{pH}$-insensitive formulation, in contrast to $\mathrm{pH}$ sensitive PE/CHEMS liposomes [11,23-25]. ProtamineDNA condensate at a $1.6 / 1(+/-)$ theoretical charge ratio

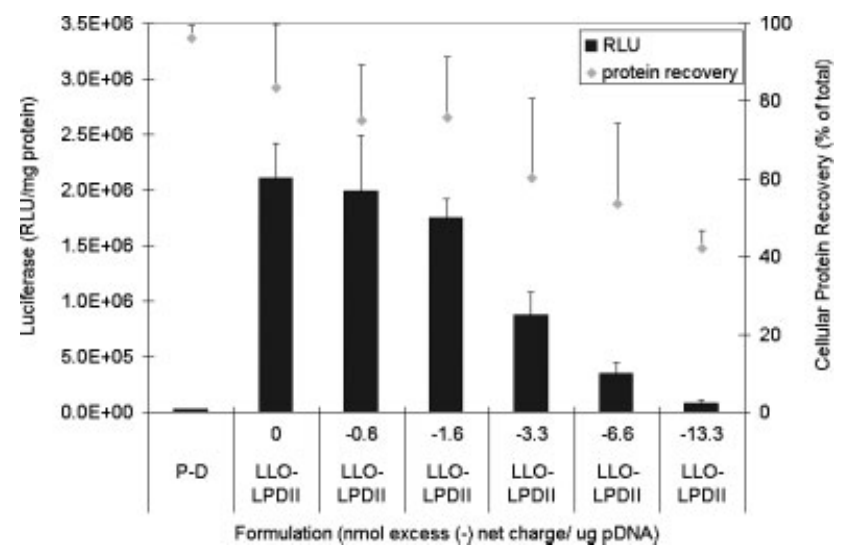

Figure 4. Effect of the total liposome content on transfection activity. pDNA was condensed using protamine to a 1.6:1 $(+/-)$ theoretical charge ratio. The cationic protamine-pDNA condensates were complexed with increasing amounts of anionic PE/CHEMS liposomes containing LLO past the charge neutralization point to include increasing excess net negative charge. pDNA condensed with protamine in the absence of liposomes was given as a control formulation (P-D). Results are reported as the mean $\pm S E M$ of three experiments performed in triplicate

was complexed with a fixed amount of excess anionic liposomes made of either PC/CHEMS or PE/CHEMS. To assess the relative effectiveness of LLO in each of the two systems, the two lipid compositions were each tested with LLO-liposomes at three ratios of blank liposomes to LLOliposomes: entirely blank liposomes (0\% LLO-liposomes), liposomes pre-mixed to include 50\% LLO-liposomes and $50 \%$ blank liposomes (50\%), and entirely LLO-containing liposomes (100\%). As shown in Figure 5, LLO was less effective at improving transfection in the $\mathrm{pH}$-insensitive system ( $p<0.05$ for PC vs. PE). While a modest increase in transfection was observed in the PC/CHEMS system upon LLO incorporation, the effect was more pronounced in the PE/CHEMS formulation, with $p<0.05$ for both $50 \%$ and $100 \%$ LLO-liposome formulations compared to formulations lacking LLO (two-way ANOVA).

\section{Effect of serum on transfection}

An important attribute of anionic liposomal delivery systems, in contrast to cationic liposomes or particulate carriers, is the potential ability to function in the presence of serum. In order to determine whether the LLO-LPDII delivery vehicle is affected by the presence of serum, we used serum-free transfection media for a comparative study. This study showed no significant difference between the presence and absence of serum during the 4-h incubation period using either protamine-DNA alone, anionic LPDII, or LLO-LPDII complexes (Figure 6). LLO was capable of mediating improved transfection in LLOLPDII over LPDII in the presence and absence of serum $(p<0.05)$. 


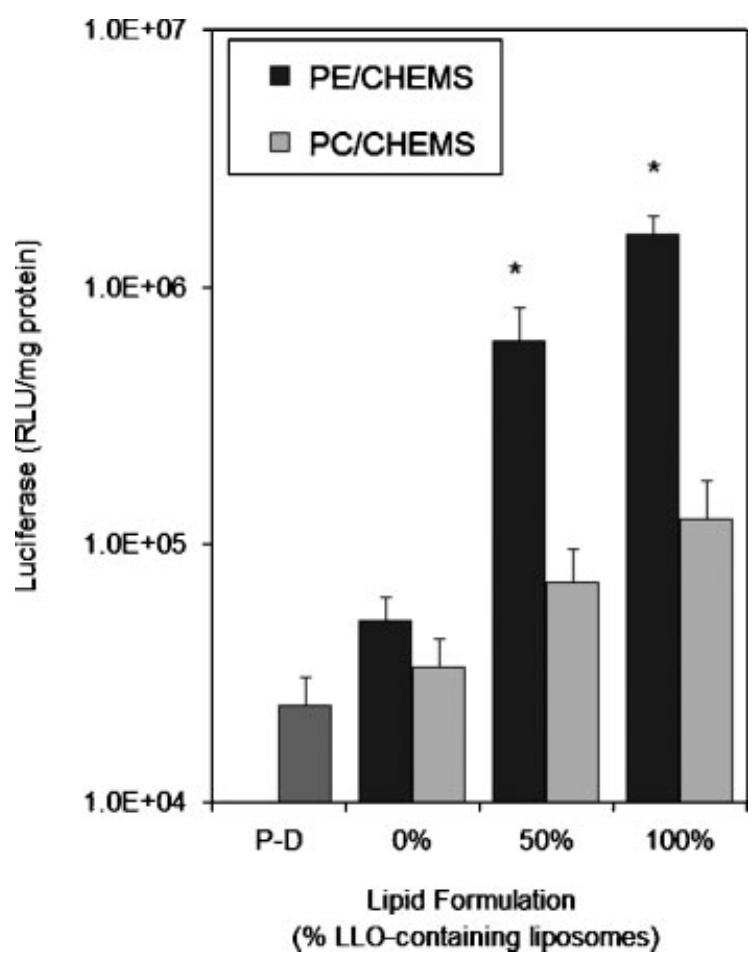

Figure 5. Effect of the pH-sensitivity of liposomes in LLO-LPDII on transfection activity. Protamine-pDNA condensates at a 1.6:1 $(+/-)$ theoretical charge ratio were complexed with anionic liposomes composed of either $\mathrm{pH}$-insensitive (PC/CHEMS) or $\mathrm{pH}$-sensitive (PE/CHEMS) lipids to a net charge of $-1.9 \mathrm{nmol} / \mu \mathrm{g}$ pDNA. The portion of each liposome formulation which was composed of LLO-containing liposomes was varied from all blank liposomes ( $0 \%$ ) to all LLO-containing liposomes (100\%). Results are reported as the mean \pm SEM of five experiments performed in triplicate

\section{Comparison with existing pDNA delivery methods}

The LPDII-LLO delivery system was compared to existing pDNA delivery technologies in order to assess the relative efficacy of this system both in the presence and absence of serum in the transfection medium (Figure 7). Chloroquine was added to the transfection media $(80 \mu \mathrm{M})$ during the 4-h transfection period in samples containing protamineDNA alone. Additionally, the well-known cationic lipoplex transfection agents Lipofectamine and DOTAP/DOPE $(1: 1)$, formulated with the same amount of pDNA, were compared to the LPDII-LLO system. In the absence of serum, the LPDII-LLO system was less effective than DOTAP/DOPE, Lipofectamine, and chloroquine-mediated transfection. However, in the presence of 5\% serum, the LPDII-LLO complex showed transfection efficiency as high as all other transfection-enhancing agents tested.

\section{Discussion}

The anionic LPDII delivery system for DNA has previously been shown to have transfection efficacy when combined with targeting modalities; therefore, we hypothesized that

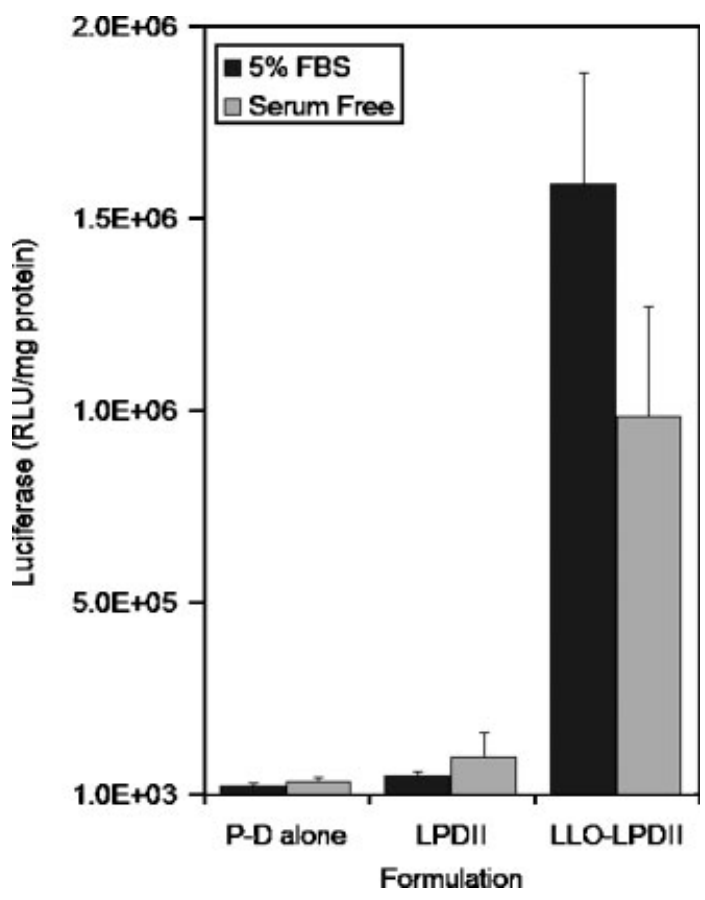

Figure 6. The effect of $5 \%$ FBS on transfection levels. pDNA was condensed with protamine sulfate at a $1.6: 1(+/-)$ charge ratio. The condensate was then complexed with either blank (LPDII) or LLO-containing PE/CHEMS liposomes (LLO-LPDII) at a $-1.9 \mathrm{nmol}$ net charge/ $\mu \mathrm{g}$ pDNA. The complexes were diluted with serum-containing transfection media as usual, or with transfection media which did not contain FBS. The final concentration of FBS in the serum-containing transfection media was $5 \%$. Results are reported as the mean \pm SEM of three experiments performed in triplicate

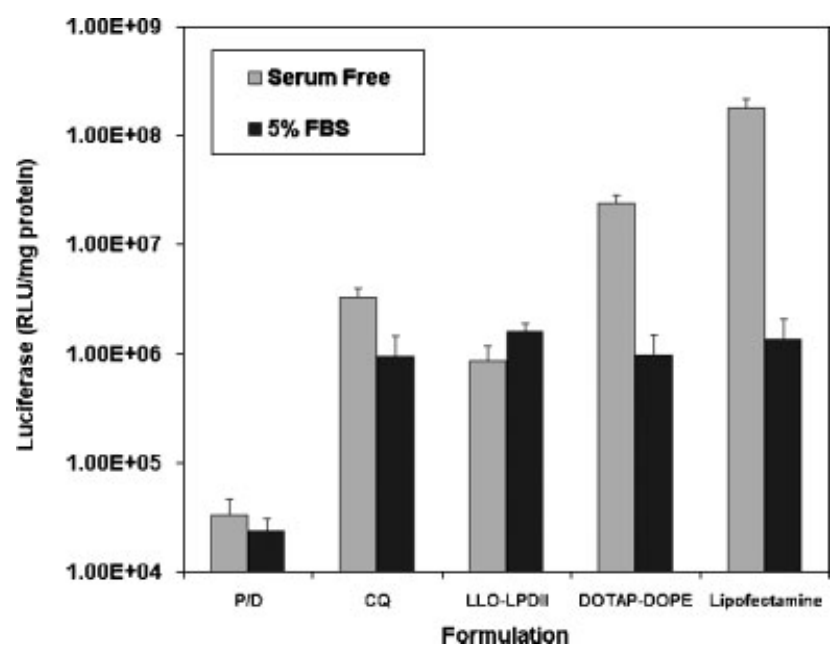

Figure 7. Comparison with common transfection agents. pDNA was condensed with protamine at a $1.6: 1(+/-)$ charge ratio for the protamine-pDNA, chloroquine (CQ), and LPDII-LLO samples, and lipid added to the appropriate samples to a net negative charge of $1.9 \mathrm{nmol} / \mu \mathrm{g}$ pDNA. pDNA was complexed directly to DOTAP/DOPE $(1: 1)$ liposomes and Lipofectamine for DOTAP/DOPE and Lipofectamine samples. pDNA ( $2 \mu \mathrm{g})$ was transfected per well under all conditions. Chloroquine was included in the indicated sample in the transfection media at $80 \mu \mathrm{M}$ during the 4-h transfection period. Results are reported as the mean \pm SEM of three experiments performed in triplicate 
the LPDII particle has the necessary versatility to provide a general scaffold onto which additional, improved functionalities can be added for pDNA delivery [10]. This study focuses on combining endosomolytic activity with the LPDII carrier through the encapsulation of LLO into the liposomal component of the system. We have incorporated LLO in the LPDII particles, characterized key parameters including the ratios of cation to pDNA, liposomes to pDNA condensate, and LLO to non-LLOliposomes, and demonstrated the effect of LLO on enhanced delivery of pDNA. The results in Figure 1 clearly show that incorporation of LLO into the LPDII system mediates higher transfection efficiency in P388D1 cells, demonstrating that LLO enables transfection of macrophages using untargeted anionic LPDII carriers. The result also shows that inactivation of the LLO prior to complex formation with pDNA-protamine condensate results in loss of this transfection enhancement, with transfection levels falling back to the levels of the LPDII system without LLO and to those of pDNA condensed with protamine alone. Thus improved transfection can be directly linked to the LLO that is active. Our data is in agreement with previous work in which anionic LPDII without targeting was not efficient in transfection and only effective when modified with targeting ligand [10]. Furthermore, addition of free LLO to transfection mixtures during formation of LPDII was unable to enhance transfection above the levels of LPDII alone. This control mimics the possible condition where LLO is released from the liposome during LLO-LPDII formation. This result demonstrates that transfection using LLO-LPDII requires encapsulation of the LLO with the delivery vehicle, suggesting that LLO encapsulated in liposomes is not released during complexation with the cationic protamine-pDNA during the formation of LLO-LPDII.

The net charge of the protamine-DNA condensate had a significant effect on transfection levels achieved by the LLO-LPDII, as seen in Figure 2, with the formation of a cationic protamine-DNA condensate being essential for LLO-LPDII effectiveness. The simplest explanation for this effect is that anionic protamine-DNA condensates do not form stable electrostatic complexes with anionic liposomes. The transfection level of the most anionic condensate $(0.64 / 1,+/-)$ was comparable to transfection with protamine-DNA in the absence of liposomes, further suggesting a lack of complex formation in the anionic condensates. The anionic condensate data points serve as both a negative control and as evidence that complexation through electrostatics is necessary.

After examining the positive trend exhibited by the inclusion of LLO-containing liposomes, we determined the effect of increasing the total amount of lipid while holding both the protamine and pDNA levels constant. However, we found an acute decrease in transfection with the presence of excess LLO-containing liposomes. One explanation for this trend is that the excess liposomes which do not complex to the protamine-DNA simply compete with the LLO-LPDII complexes and reduce either the binding or uptake of the complexes. The data in
Figure 4 indicate that the LLO-LPDII complex is relatively sensitive to the total liposome content in vitro. This trend might not be important in vivo, where excess liposomes would be unlikely to exert a significant competitive effect at a specific cell target. Additionally, some toxicity was demonstrated at high levels of LLO-containing liposome excess, as determined by total cellular recovery measurements, which may also have contributed to lower transfection levels. It is important to note, however, that all luciferase assay results were normalized for cellular protein recovery, and thus any results strictly attributed to lower cell numbers were corrected for.

Our data verify that $\mathrm{pH}$-sensitivity is critical for successful transfection using the LLO-LPDII complex. The data in Figure 5 are consistent with the hypothesis that LLO must be released from its liposomal encapsulation in order to facilitate delivery of the condensed plasmid pDNA from the endosome into the cytosol, similar to the results for the delivery of other macromolecules $[11,17]$. This data suggests that the transfection utilizing LLO-LPDII is largely dependent on destabilization of the liposomal carrier in the endosome, making $\mathrm{pH}$-sensitive liposomes a critical component of the LLO-LPDII system through facilitating improved transfection in the LLOLPDII system, consistent with endosomal escape and subsequent cytosolic delivery as the route of delivery.

Protamine-DNA alone, LPDII particles, and LLO-LPDII complexes transfected cells similarly in the presence and absence of 5\% FBS (Figure 6), demonstrating that these anionic LLO-LPDII particles are serumcompatible. As serum is unavoidable in vivo, such compatibility is important in gene delivery carrier development. Furthermore, this result is in contrast with cationic delivery vehicles which tend to decrease their effectiveness in the presence of serum [4,26,27].

To gauge the efficacy of this modified LLO-LPDII system, we compared the transfection levels of the complex in the presence and absence of 5\% serum with several commonly used in vitro transfection agents: chloroquine, DOTAP/DOPE cationic liposomes, and Lipofectamine (Figure 7). In the presence of 5\% FBS in the transfection media, the LLO-LPDII particle showed transfection levels comparable to both cationic lipid formulations and chloroquine-mediated transfection. The ability of the LLOLPDII particle to maintain its transfection levels in the presence of serum at a level that is comparable to these common transfection agents shows its promise as a serumcompatible delivery vehicle, though further enhancement of this system through additional modifications such as targeting may allow for even higher transfection levels.

Methods to overcome the series of barriers to DNA delivery must continue to be developed. LLO-LPDII has been shown here to enhance transfection levels significantly over its anionic LPDII predecessor, and additionally it maintains the ability to be targeted. The combination of the two concepts, improved uptake due to targeting and enhanced cytosolic delivery by LLO incorporation, would effectively surpass two major barriers to pDNA delivery at the level of the cell. 
Additionally, although not tested in this report, the use of protamine sulfate may facilitate localization to the nucleus through its NLS-like sequences [28]. As there is a potential drug delivery loss at any barrier to delivery, systems which combine different delivery methods are most likely to ultimately enhance gene delivery to adequate levels for vaccine delivery or gene therapy. This paper demonstrates the successful combination of two technologies, the LPDII anionic liposome system and LLO, an endosomolytic poreforming protein. Conceptualization and validation of the LLO-LPDII hybrid system represents an important step toward efficiently expanding the scope of gene delivery applications.

\section{Acknowledgements}

The authors wish to thank Dr. Daniel A. Portnoy for the gift of his-LLO and Dr. Gary Nabel for the gift of pNGVL3. This work was supported by the National Institutes of Health (grant R01AI47173 to K.-D. L.) and partially by the Vahlteich Research Fund at the University of Michigan. G. Lorenzi was supported by fellowships from the Fannie and John Hertz Foundation, the University of Michigan, and AFPE.

\section{References}

1. Christ M. Preclinical evaluation of gene transfer products: safety and immunological aspects. Toxicology 2002; 174: 13-19.

2. Robbins PD, Tahara H, Ghivizzani SC. Viral vectors for gene therapy. Trends Biotechnol 1998; 16: 35-40.

3. Ledley F. Nonviral gene therapy: the promise of genes as pharmaceutical products. Hum Gene Ther 1995; 6: 1129-1144.

4. Nishikawa M, Huang L. Nonviral vectors in the new millennium: delivery barriers in gene transfer. Hum Gene Ther 2001; 12: $861-870$.

5. Thomas CE, Ehrhardt A, Kay MA. Progress and problems with the use of viral vectors for gene therapy. Nat Rev Genet 2003; 4: $346-358$

6. Pouton CW, Seymour LW. Key issues in non-viral gene delivery. Adv Drug Deliv Rev 2001; 46: 187-203.

7. Wagner E. Application of membrane-active peptides for nonviral gene delivery. Adv Drug Deliv Rev 1999; 38: 279-289.

8. Larson GM, Lee K-D. Macromolecular cytosolic delivery: cell membranes as the primary obstacle. Arch Pharm Res 1998; 21: 621-628.

9. Gottschalk S, Tweten R, Smith L, et al. Efficient gene delivery and expression in mammalian cells using DNA coupled with perfringolysin O. Gene Ther 1995; 2: 498-503.

10. Lee R, Huang L. Folate-targeted, anionic liposome-entrapped polylysine-condensed DNA for tumor cell-specific gene transfer. J Biol Chem 1996; 271: 8481-8487.
11. Lee K-D, Oh Y, Portnoy D, et al. Delivery of macromolecules into cytosol using liposomes containing hemolysin from Listeria monocytogenes. J Biol Chem 1996; 271: 7249-7252.

12. Dramsi S, Cossart P. Listeriolysin O: a genuine cytolysin optimized for an intracellular parasite. J Cell Biol 2002; 156: 943-946.

13. Bielecki J, Youngman P, Connelly P, et al. Bacillus subtilis expressing a hemolysin gene from Listeria monocytogenes can grow in mammalian cells. Nature 1990; 345: 175-176.

14. Mandal M, Lee KD. Listeriolysin O-liposome-mediated cytosolic delivery of macromolecule antigen in vivo: enhancement of antigen-specific cytotoxic T lymphocyte frequency, activity, and tumor protection. Biochim Biophys Acta 2002; 1563: 7-17.

15. Mathew E, Hardee GE, Bennett CF, et al. Cytosolic delivery of antisense oligonucleotides by listeriolysin O-containing liposomes. Gene Ther 2003; 10: 1105-1115.

16. Li S, Huang L. In vivo gene transfer via intravenous administration of cationic lipid-protamine-DNA (LPD) complexes. Gene Ther 1997; 4: 891-900.

17. Litzinger DC, Huang L. Phosphatidylethanolamine liposomes: drug delivery, gene transfer and immunodiagnostic applications. Biochim Biophys Acta 1992; 1113: 201-227.

18. Gedde MM, Higgins DE, Tilney LG, et al. Role of listeriolysin O in cell-to-cell spread of Listeria monocytogenes. Infect Immunol 2000; 68: 999-1003.

19. Saito G, Amidon GL, Lee KD. Enhanced cytosolic delivery of plasmid DNA by a sulfhydryl-activatable listeriolysin $\mathrm{O} /$ protamine conjugate utilizing cellular reducing potential. Gene Ther 2003; 10: 72-83.

20. Papahadjopoulos D, Allen TM, Gabizon A, et al. Sterically stabilized liposomes: improvements in pharmacokinetics and antitumor therapeutic efficacy. Proc Natl Acad Sci U S A 1991; 88: $11460-11464$.

21. Drummond DC, Meyer O, Hong K, et al. Optimizing liposomes for delivery of chemotherapeutic agents to solid tumors. Pharmacol Rev 1999; 51: 691-743.

22. Tighe H, Corr M, Roman M, et al. Gene vaccination: plasmid DNA is more than just a blueprint. Immunol Today 1998; 19: 89-97.

23. Straubinger RM. pH-sensitive liposomes for delivery of macromolecules into cytoplasm of cultured cells. Methods Enzymol 1993; 221: 361-376.

24. Düzgünes N, Straubinger RM, Baldwin PA, et al. Proton-induced fusion of oleic acid-phosphatidylethanolamine liposomes. Biochemistry 1985; 24: 3091-3098.

25. Connor J, Huang L. Efficient cytoplasmic delivery of a fluorescent dye by $\mathrm{pH}$-sensitive immunoliposomes. J Cell Biol 1985; 101: 582-589.

26. Reimer D, Zhang Y, Kong S, et al. Formation of novel hydrophobic complexes between cationic lipids and plasmid DNA. Biochemistry 1995; 34: 12 877-12883.

27. Ahsan F, Rivas IP, Khan MA, et al. Targeting to macrophages: role of physicochemical properties of particulate carriers - liposomes and microspheres - on the phagocytosis by macrophages. $J$ Control Release 2002; 79: 29-40.

28. Sorgi FL, Bhattacharya S, Huang L. Protamine sulfate enhances lipid-mediated gene transfer. Gene Ther 1997; 4: 961-968. 